MATHEMATICS OF COMPUTATION

Volume 75, Number 254, Pages 891-902

S 0025-5718(05)01803-X

Article electronically published on November 30, 2005

\title{
LINEAR LAW FOR THE LOGARITHMS OF THE RIEMANN PERIODS AT SIMPLE CRITICAL ZETA ZEROS
}

\author{
KEVIN A. BROUGHAN AND A. ROSS BARNETT
}

\begin{abstract}
Each simple zero $\frac{1}{2}+i \gamma_{n}$ of the Riemann zeta function on the critical line with $\gamma_{n}>0$ is a center for the flow $\dot{s}=\xi(s)$ of the Riemann xi function with an associated period $T_{n}$. It is shown that, as $\gamma_{n} \rightarrow \infty$,

$$
\log T_{n} \geq \frac{\pi}{4} \gamma_{n}+O\left(\log \gamma_{n}\right) .
$$

Numerical evaluation leads to the conjecture that this inequality can be replaced by an equality. Assuming the Riemann Hypothesis and a zeta zero separation conjecture $\gamma_{n+1}-\gamma_{n} \gg \gamma_{n}^{-\theta}$ for some exponent $\theta>0$, we obtain the upper bound $\log T_{n} \ll \gamma_{n}^{2+\theta}$. Assuming a weakened form of a conjecture of Gonek, giving a bound for the reciprocal of the derivative of zeta at each zero, we obtain the expected upper bound for the periods so, conditionally, $\log T_{n}=\frac{\pi}{4} \gamma_{n}+O\left(\log \gamma_{n}\right)$. Indeed, this linear relationship is equivalent to the given weakened conjecture, which implies the zero separation conjecture, provided the exponent is sufficiently large. The frequencies corresponding to the periods relate to natural eigenvalues for the Hilbert-Polya conjecture. They may provide a goal for those seeking a self-adjoint operator related to the Riemann hypothesis.
\end{abstract}

\section{INTRODUCTION}

If a holomorphic function of a single complex variable $f(s)$ has a simple zero at $s_{o}$ which is a center for the dynamical system $\dot{s}=f(s)$, then the period of an orbit encircling $s_{o}$ is given by $2 \pi i / f^{\prime}\left(s_{o}\right)$ [3. Theorem 2.3], and, in particular, is independent of the orbit. When this is applied to the simple zeros of Riemann's function $\xi(s)$, which lie on the critical line $s=\frac{1}{2}$ and which coincide with the simple critical zeros of $\zeta(s)$, we see that the periods $\left\{\tau_{n}=2 \pi i / \xi^{\prime}\left(\frac{1}{2}+i \gamma_{n}\right): \xi\left(\frac{1}{2}+i \gamma_{n}\right)=0\right\}$ could be of interest since each such (real) number applies to an infinite family of nested orbits. See Figure 1 where selected periodic orbits surrounding the 27 th and 28 th zeros of zeta for the flow $\dot{s}=\xi(s)$ are displayed.

Even though the positions of the zeros on the critical line have a considerable degree of random variation, the values of the periods are quite constrained, as is illustrated numerically in Figure 2 and partly proved in Theorem 4.1: The logarithm of the absolute value of the periods varies linearly with the position of the zero.

Received by the editor December 13, 2004 and, in revised form, March 17, 2005.

2000 Mathematics Subject Classification. Primary 11M06, 11M26, 11S40.

Key words and phrases. Riemann zeta function, xi function, zeta zeros, periods, critical line, Hilbert-Polya conjecture. 


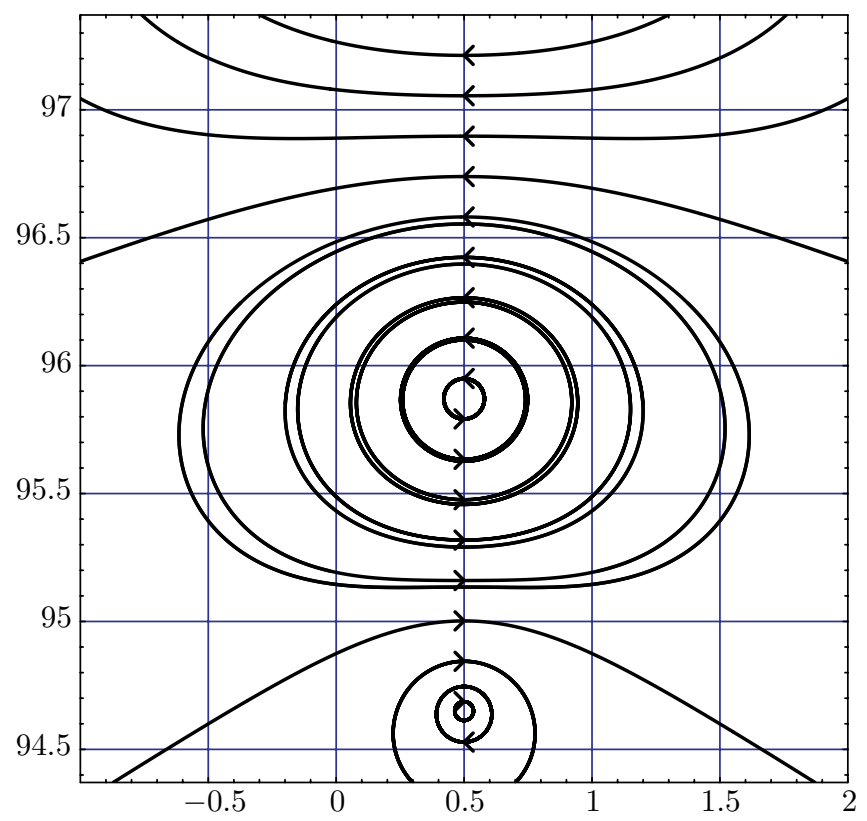

FiguRe 1 . Orbits around the 27 th and 28 th zero for $\dot{z}=\xi(z)$.

In Section 2 the numerical evaluation is developed. Of the 500 zeta zeros studied, being those with smallest positive $t$ coordinates, the periods always increased with increasing zero value, with four exceptions. The numerical evidence demonstrates a relationship between each period and the $t$-coordinate of the corresponding zero which is very close to being linear.

In Section 3 some preliminary lemmas are given.

In Section 4 the relationship

$$
\log T_{n} \geq \frac{\pi}{4} \gamma_{n}+O\left(\log \gamma_{n}\right)
$$

where $T_{n}=(-1)^{n} \tau_{n}$ is the absolute value of the period, is proved. An upper bound is derived subject to the Riemann Hypothesis and a plausible conjecture on zero separations. Using a weakened form of a conjecture of Gonek (which includes the Riemann Hypothesis), namely, that there exists a nonnegative real number $\theta$ such that $\left|\zeta^{\prime}\left(\frac{1}{2}+i \gamma_{n}\right)\right|^{-1}=O\left(\left|\gamma_{n}\right|^{\theta}\right)$ for all $n \in \mathbb{N}$, we derive the precise upper bound and hence, conditionally,

$$
\log T_{n}=\frac{\pi}{4} \gamma_{n}+O\left(\log \gamma_{n}\right)
$$

In Section 5 a possible significance of the periods, in the context of the HilbertPolya conjecture, is sketched.

We first define some notations. Let $s=\sigma+i t, \xi(s)=u(\sigma, t)+i v(\sigma, t)$. Label the simple zeros of $\xi(s)$, with a positive $t$ coordinate, going up the critical line as $\left\{\gamma_{n}: n \in \mathbb{N}\right\}$. A particular zero $\frac{1}{2}+i \gamma_{n}$ has the corresponding period

$$
\tau_{n}=\frac{2 \pi i}{\xi^{\prime}\left(\frac{1}{2}+i \gamma_{n}\right)} \text {. }
$$


Because $\xi(s)$ is real on the critical line, $v_{t}\left(\frac{1}{2}, t\right)=0$, so $\xi^{\prime}\left(\frac{1}{2}+i t\right)=-i u_{t}\left(\frac{1}{2}, t\right)$. Therefore $T_{n}=2 \pi /\left|u_{t}\left(\frac{1}{2}, \gamma_{n}\right)\right|$.

Let $s=\frac{1}{2}+i t$ and write the $\xi$ definition from Section 2 as

$$
\begin{aligned}
\xi\left(\frac{1}{2}+i t\right) & =e^{\log \Gamma\left(\frac{s}{2}\right)} \pi^{-s / 2} \frac{s(s-1)}{2} \zeta(s) \\
& =-e^{\Re \log \Gamma\left(\frac{s}{2}\right)} \pi^{-1 / 4}\left(\frac{t^{2}+1 / 4}{2}\right) \times Z(t) \\
& =Y(t) \times Z(t),
\end{aligned}
$$

where

$$
\begin{aligned}
\log \Gamma(s+1) & =\left(s+\frac{1}{2}\right) \log s-s+c_{1}+O\left(\frac{1}{s}\right) \\
Z(t) & =e^{i \vartheta(t)} \zeta\left(\frac{1}{2}+i t\right) \\
\vartheta(t) & =\frac{t}{2} \log \left(\frac{t}{2 \pi}\right)-\frac{t}{2}-\frac{\pi}{8}+O\left(\frac{1}{t}\right),
\end{aligned}
$$

and where here and in what follows the $c_{1}, c_{2}, \ldots$ are absolute constants.

\section{Numerical evaluation of the Periods}

We used the Chebyshev method of P. Borwein [2] to evaluate $\zeta(s)$. Our version is described in [3, section 5] in which accuracies for the $\zeta$-zeros $\gamma_{1}-\gamma_{502}$ better than $10^{-10}$ were demonstrated.

A program was written in MATLAB using the methods of Godfrey [10] for both the $\zeta$-function and the complex $\Gamma$-function. They are available in Godfrey's MATLAB site as part of the zeta-function code. In particular, values of the gamma

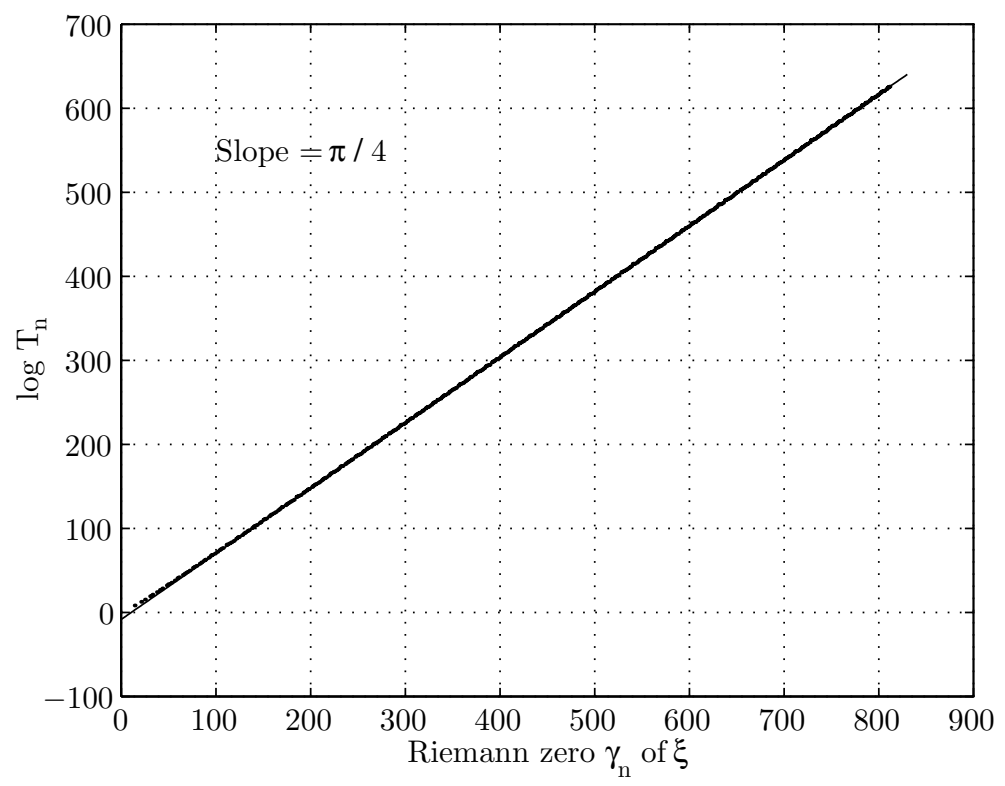

FiguRE 2. Plot of logarithm of the period magnitudes against the Riemann zeros up to $\gamma_{502}=814.1 \cdots$. 
TABLE 1. Numerical values of the first $20 \gamma_{n}$-values and logarithms of the periods.

\begin{tabular}{|c|c|c|c|}
\hline$n$ & $\gamma_{n}$ & $\log T_{n}$ & $T_{n}$ \\
\hline 1 & 14.13473 & 8.4242 & $4.5562 \mathrm{e}+003$ \\
\hline 2 & 21.02204 & 12.7793 & $3.5480 \mathrm{e}+005$ \\
\hline 3 & 25.01086 & 15.4167 & $4.9589 \mathrm{e}+006$ \\
\hline 4 & 30.42488 & 19.3796 & $2.6089 \mathrm{e}+008$ \\
\hline 5 & 32.93506 & 21.1554 & $1.5406 \mathrm{e}+009$ \\
\hline 6 & 37.58618 & 24.2391 & $3.3645 \mathrm{e}+010$ \\
\hline 7 & 40.91872 & 26.9664 & $5.1445 \mathrm{e}+011$ \\
\hline 8 & 43.32707 & 28.5531 & $2.5145 \mathrm{e}+012$ \\
\hline 9 & 48.00515 & 32.2017 & $9.6613 \mathrm{e}+013$ \\
\hline 10 & 49.77383 & 33.6324 & $4.0399 \mathrm{e}+014$ \\
\hline 11 & 52.97032 & 35.4970 & $2.6071 \mathrm{e}+015$ \\
\hline 12 & 56.44625 & 38.1368 & $3.6525 \mathrm{e}+016$ \\
\hline 13 & 59.34704 & 40.8634 & $5.5814 \mathrm{e}+017$ \\
\hline 14 & 60.83178 & 41.8135 & $1.4434 \mathrm{e}+018$ \\
\hline 15 & 65.11254 & 44.7276 & $2.6604 \mathrm{e}+019$ \\
\hline 16 & 67.07981 & 46.4654 & $1.5124 \mathrm{e}+020$ \\
\hline 17 & 69.54640 & 48.1391 & $8.0639 \mathrm{e}+020$ \\
\hline 18 & 72.06716 & 49.7490 & $4.0339 \mathrm{e}+021$ \\
\hline 19 & 75.70469 & 53.0341 & $1.0775 \mathrm{e}+023$ \\
\hline 20 & 77.14484 & 54.3339 & $3.9529 \mathrm{e}+023$ \\
\hline
\end{tabular}

function needed for the definition

$$
\xi(s)=\frac{s}{2}(s-1) \Gamma\left(\frac{s}{2}\right) \pi^{-s / 2} \zeta(s),
$$

TABLE 2. Numerical values of the logarithms of the periods and $\gamma_{n}$-values showing the four anomalous, decreasing periods as $n$ increases.

\begin{tabular}{|c|c|c|c|}
\hline$n$ & $\gamma_{n}$ & $\log T_{n}$ & $T_{n}$ \\
\hline 299 & 540.63139 & 415.2032 & $2.0914 \mathrm{e}+180$ \\
\hline 300 & 541.84744 & 414.7731 & $1.3605 \mathrm{e}+180$ \\
\hline 301 & 544.32389 & 416.2643 & $6.0437 \mathrm{e}+180$ \\
\hline \hline 364 & 630.80578 & 485.7808 & $9.3738 \mathrm{e}+210$ \\
\hline 365 & 632.22514 & 485.6408 & $8.1491 \mathrm{e}+210$ \\
\hline 366 & 633.54686 & 486.1135 & $1.3075 \mathrm{e}+211$ \\
\hline \hline 446 & 740.57381 & 571.0652 & $1.0244 \mathrm{e}+248$ \\
\hline 447 & 741.75734 & 571.0002 & $9.5995 \mathrm{e}+247$ \\
\hline 448 & 743.89501 & 572.2458 & $3.3357 \mathrm{e}+248$ \\
\hline \hline 481 & 786.46115 & 606.9047 & $3.7614 \mathrm{e}+263$ \\
\hline 482 & 787.46846 & 606.8254 & $3.4747 \mathrm{e}+263$ \\
\hline 483 & 790.05909 & 608.9406 & $2.8810 \mathrm{e}+264$ \\
\hline
\end{tabular}




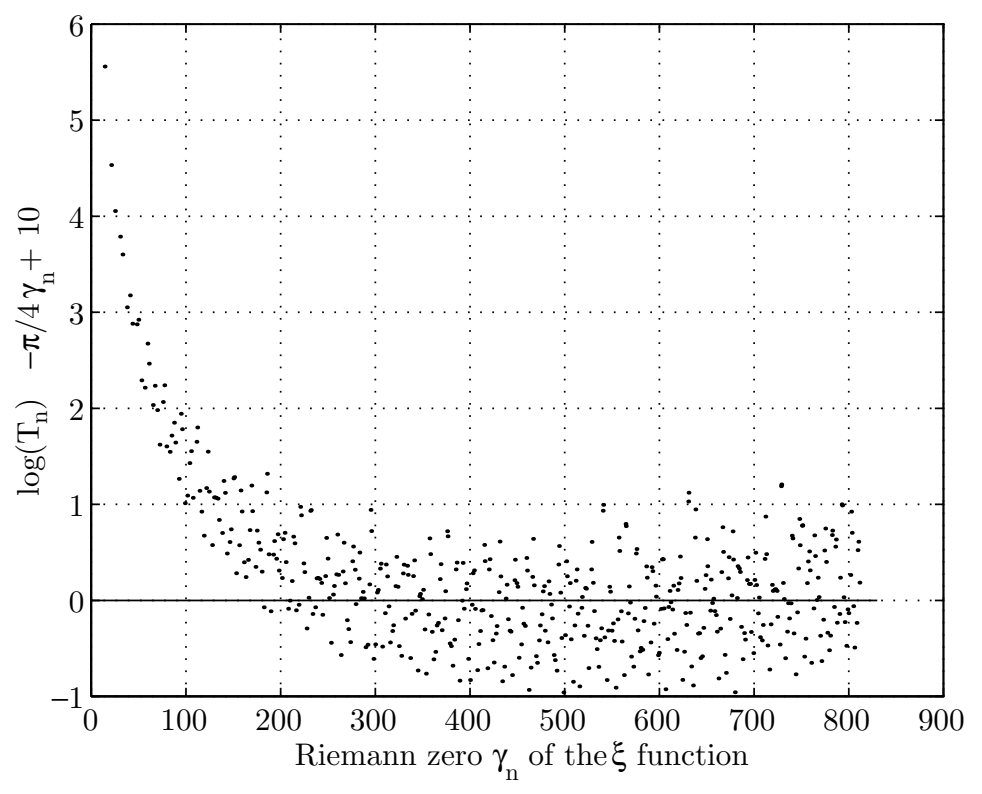

Figure 3 . The residuals using a slope of $\pi / 4$.

do not have to be incorporated into a Riemann-Siegel formulation. The values $\xi^{\prime}\left(\frac{1}{2}+i \gamma_{n}\right)$ were found by differencing. Figure 2 gives an illustration of the linear relation of this paper for zeros up to $t=\gamma_{502}=814.1 \cdots$.
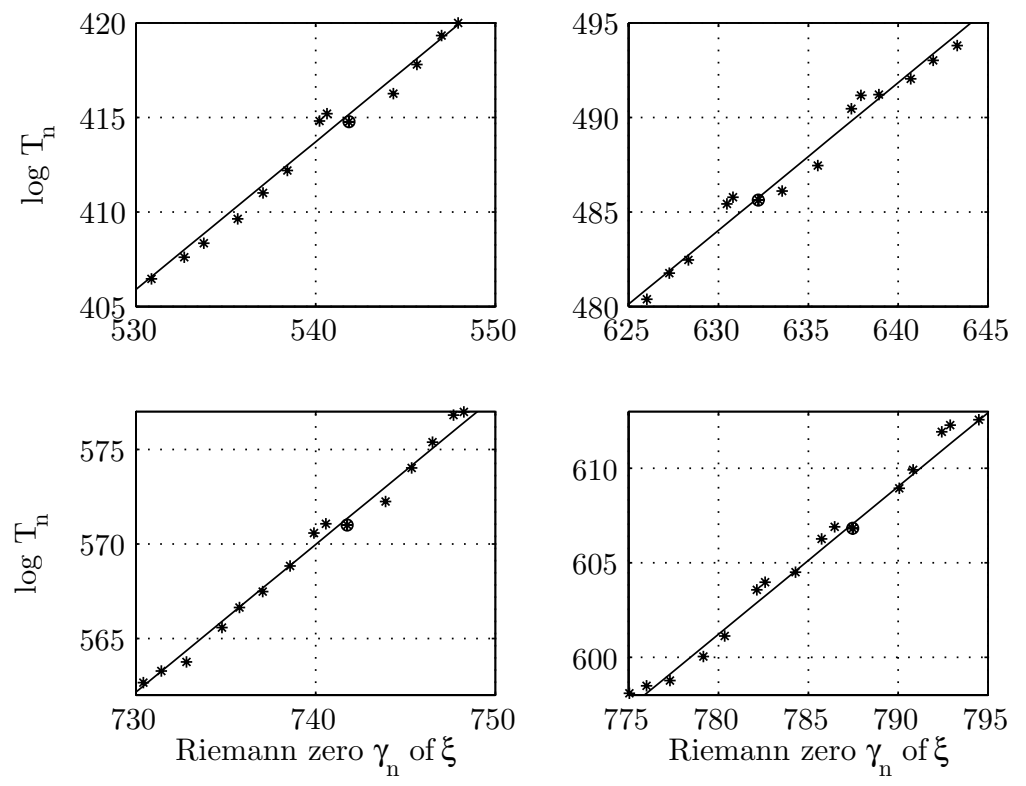

Figure 4. The first four anomalous deviations from monotonicity (circled). 

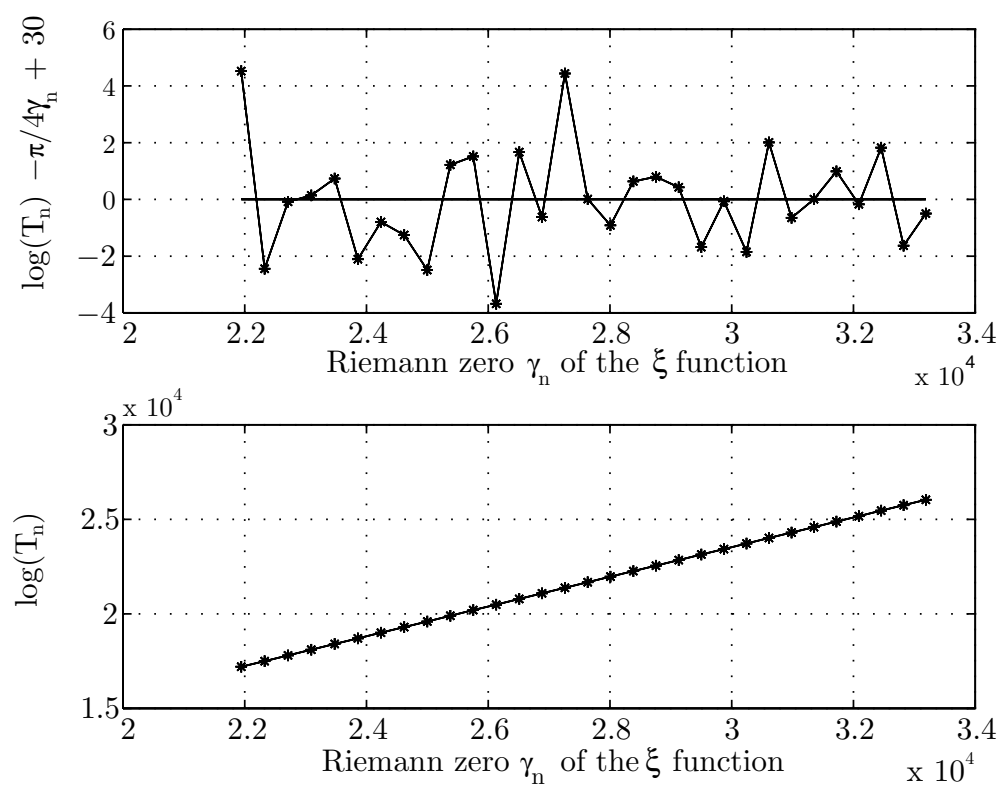

FiguRE 5. The linear periods (below) and residuals (above) using a slope of $\pi / 4$.

Figure 3 shows the deviations from the linear law $\log T_{n}=\alpha+\frac{\pi}{4} \gamma_{n}$. There is a rise, matching an exponential, at low $\gamma$-values. The value of $\alpha$ for the fit is -10.0 .

Table 1 shows the zeros and periods for $0<n \leq 20$, where the slope deviates slightly from $\pi / 4$, as is seen in Figure 3 . The rms deviation for the complete fit is 1.5 .

Table 2 includes the zeros and periods for $\gamma_{300}=541.847 \cdots, \gamma_{365}=632.225 \cdots$, $\gamma_{447}=741.757 \cdots$, and $\gamma_{482}=787.468 \cdots$, where, anomalously, we find $\log T_{n}<$ $\log T_{n-1}$ which are the only examples below $\gamma_{502}$ where the period decreases. These are plotted with an expanded scale in Figure 4. An alternative calculation was devised to extend the modest upper value of $t \sim 800$ to $t \sim 34000$. The same linear relationship was found to hold. The symbolic $\zeta$-calculations of Maple ${ }^{T M}$ were combined with Godfrey's implementation of Lanczos' $\Gamma$-function method. Earlier values $t \leq 814.1 \cdots$ were confirmed. The results are presented in increments of 500 in Figure 5.

\section{Preliminary lemmas}

Lemma 3.1. As $t \rightarrow \infty$

$$
\Re \log \Gamma\left(\frac{\frac{1}{2}+i t}{2}\right)=-\frac{1}{8} \log \left(9+4 t^{2}\right)-\frac{\pi}{4} t+c_{2}+O\left(\frac{1}{t}\right) .
$$

Proof. This follows directly from Stirling's approximation given in Section 1 above.

Lemma 3.2. As $t \rightarrow \infty,\left|\vartheta^{\prime}(t)\right|=O(\log t)$.

Proof. This follows directly from the expression for $\vartheta(t)$ given above or see [8, Page 125]. 
Lemma 3.3. As $t \rightarrow \infty,\left|\zeta\left(\frac{1}{2}+i t\right)\right|=O\left(t^{1 / 6} \log ^{3 / 2} t\right)$.

Proof. This is [17, Page 99].

Lemma 3.4. As $t \rightarrow \infty$,

$$
\begin{aligned}
\left|\zeta^{\prime}\left(\frac{1}{2}+i t\right)\right| & =O\left(t^{1 / 4} \log ^{2} t\right) \\
\text { and }\left|\zeta^{\prime \prime}\left(\frac{1}{2}+i t\right)\right| & =O\left(t^{1 / 4} \log ^{4} t\right) .
\end{aligned}
$$

Proof. These bounds follow from the upper bound for $\zeta^{(k)}\left(\frac{1}{2}+i t\right)$, derived using the approximate functional equation and Cauchy's integral formula for the $n$th derivative given in [13, Page 57], namely,

$$
\begin{aligned}
\left|\zeta^{(k)}\left(\frac{1}{2}+i t\right)\right| \leq & \left|\sum_{n \leq \sqrt{t / 2 \pi}} \frac{\log ^{k} n}{n^{1 / 2+i t}}\right|+\log ^{k} t \sum_{1 \leq j \leq k}\left|\sum_{n \leq \sqrt{t / 2 \pi}} \frac{\log ^{j} n}{n^{1 / 2-i t}}\right| \\
& +O\left(t^{-1 / 4} \log ^{k} t\right) .
\end{aligned}
$$

Lemma 3.5. As $t \rightarrow \infty, Z^{\prime}(t)=O\left(t^{1 / 4} \log ^{2} t\right)$.

Proof. This follows directly from $Z(t)=e^{i \vartheta(t)} \zeta\left(\frac{1}{2}+i t\right)$ after differentiating and using Lemmas 3.2 and 3.4.

\section{LOWER AND UPPER BOUNDS FOR THE LINEAR RELATIONSHIP}

Theorem 4.1. For $n \in \mathbb{N}$,

$$
\log T_{n} \geq \frac{\pi}{4} \gamma_{n}+O\left(\log \gamma_{n}\right)
$$

Proof. We first have

$$
\left|u_{t}\left(\frac{1}{2}, t\right)\right|=\left|\xi^{\prime}\left(\frac{1}{2}+i t\right)\right|
$$

and

$$
i \xi^{\prime}\left(\frac{1}{2}+i t\right)=Y^{\prime}(t) Z(t)+Y(t) Z^{\prime}(t) .
$$

Since $Z\left(\gamma_{n}\right)=0$,

$$
\left|\xi^{\prime}\left(\frac{1}{2}+i \gamma_{n}\right)\right|=\left|Y\left(\gamma_{n}\right)\right| \times\left|Z^{\prime}\left(\gamma_{n}\right)\right| .
$$

So

$$
\log \left|u_{t}\left(\frac{1}{2}, \gamma_{n}\right)\right|=\log \left|Y\left(\gamma_{n}\right)\right|+\log \left|Z^{\prime}\left(\gamma_{n}\right)\right| .
$$

By Lemma 3.1,

$$
\begin{aligned}
\log |Y(t)| & =\Re \log \Gamma\left(\frac{\frac{1}{2}+i t}{2}\right)-\frac{1}{4} \log \pi+\log \left(\frac{1}{8}+\frac{t^{2}}{2}\right) \\
& =-\frac{\pi}{4} t+\log \left(\frac{1+4 t^{2}}{\left(9+4 t^{2}\right)^{1 / 8}}\right)+c_{3}+O\left(\frac{1}{t}\right) .
\end{aligned}
$$

Therefore, by Lemma 3.5,

$$
\log \left|u_{t}\left(\frac{1}{2}, \gamma_{n}\right)\right| \leq-\frac{\pi}{4} \gamma_{n}+O\left(\log \gamma_{n}\right),
$$

and therefore

$$
\log T_{n} \geq \frac{\pi}{4} \gamma_{n}+O\left(\log \gamma_{n}\right) .
$$


Finding upper bounds for the periods appears to be a much more difficult problem. Assuming the Riemann Hypothesis (RH) and a plausible zero separation hypothesis, we make some progress on this issue. Later we show how the upper bound follows (indeed is equivalent to) a weakened form of a conjecture of Gonek.

Fujii 9] has shown that there exists a $\lambda>1$ such that for $n$ in a set of positive density

$$
\frac{\gamma_{n+1}-\gamma_{n}}{2 \pi / \log \gamma_{n}} \geq \lambda
$$

Since [17, Page 214] $\gamma_{n} \sim 2 \pi n / \log n$, Fujii's result implies for all $\epsilon>0$ and $n$ sufficiently large and in a subset of positive density that $\gamma_{n+1}-\gamma_{n} \geq 1 / \gamma_{n}^{\epsilon}$. Here we assume that for some $\theta>0$ for all $n \in \mathbb{N}$

$$
\gamma_{n+1}-\gamma_{n} \gg \frac{1}{\gamma_{n}^{\theta}},
$$

where the implied constant depends on $\theta$, but is absolute once $\theta$ has a numerical value. Call this assumption the "zero separation conjecture" or ZSC. Of course, if it is true, it implies all zeros are simple. It supports the notion of Montgomery that the zeros tend to "repel", but does allow zeros to be very close. In [11] Gonek suggests $\theta=1 / 3+\epsilon$ might be the optimal choice.

Theorem 4.2. Assume $R H$ and ZSC. Then for all $n \in \mathbb{N}$,

$$
\log T_{n} \ll \gamma_{n}^{2+\theta} .
$$

Proof. Using the shifted product representation for $\xi(s)$,

$$
\xi\left(z+\frac{1}{2}\right)=\xi\left(\frac{1}{2}\right) \prod_{n \in \mathbb{N}}\left(1+\frac{z^{2}}{\gamma_{n}^{2}}\right)
$$

leads to the value of the derivative at a zero,

$$
\begin{aligned}
u_{t}\left(\frac{1}{2}, \gamma_{n}\right) & =\frac{2 \xi\left(\frac{1}{2}\right)}{\gamma_{n}} \prod_{j \in \mathbb{N}, j \neq n}\left(1-\frac{\gamma_{n}^{2}}{\gamma_{j}^{2}}\right) \\
\left|u_{t}\left(\frac{1}{2}, \gamma_{n}\right)\right| & =\frac{2 \xi\left(\frac{1}{2}\right)}{\gamma_{n}} \prod_{1 \leq j<n}\left(\frac{\gamma_{n}^{2}}{\gamma_{j}^{2}}-1\right) \prod_{n<j}\left(1-\frac{\gamma_{n}^{2}}{\gamma_{j}^{2}}\right) \\
& =\frac{2 \xi\left(\frac{1}{2}\right)}{\gamma_{n}} X_{n} \times Y_{n}, \text { say. }
\end{aligned}
$$

Because the number of zeros up to level $T$ is less than $T \log T / 2 \pi$, it follows that

$$
X_{n}=\prod_{1 \leq j<n}\left(\frac{\gamma_{n}+\gamma_{j}}{\gamma_{j}}\right)\left(\frac{\gamma_{n}-\gamma_{j}}{\gamma_{j}}\right) \geq\left(\frac{\gamma_{n}-\gamma_{n-1}}{\gamma_{n}}\right)^{\gamma_{n} \log \gamma_{n}} .
$$

Therefore, by the assumption ZSC,

$$
-\log X_{n} \ll(\theta+1) \gamma_{n} \log ^{2} \gamma_{n} .
$$

Also, because $-\log (1-x) \leq x /(1-x)$ for $0 \leq x<1$ and

$$
\begin{gathered}
Y_{n}=\prod_{j>n}\left(1-\frac{\gamma_{n}^{2}}{\gamma_{j}^{2}}\right), \text { then } \\
-\log Y_{n}=\sum_{j>n}-\log \left(1-\frac{\gamma_{n}^{2}}{\gamma_{j}^{2}}\right) \leq \sum_{j>n} \frac{\gamma_{n}^{2}}{\gamma_{j}^{2}-\gamma_{n}^{2}} .
\end{gathered}
$$


But

$$
\begin{aligned}
\sum_{j>n} \frac{1}{\gamma_{j}^{2}-\gamma_{n}^{2}} & =\sum_{\gamma_{j}^{2}<\frac{10}{9} \gamma_{n}^{2}} \frac{1}{\gamma_{j}^{2}-\gamma_{n}^{2}}+\sum_{\gamma_{j}^{2} \geq \frac{10}{9} \gamma_{n}^{2}} \frac{1}{\gamma_{j}^{2}-\gamma_{n}^{2}} \\
& =S_{1}+S_{2}, \text { say. }
\end{aligned}
$$

Since, in $S_{2}, \gamma_{j}^{2}-\gamma_{n}^{2} \geq \gamma_{j}^{2}-\frac{9}{10} \gamma_{j}^{2}=\frac{1}{10} \gamma_{j}^{2}$,

$$
S_{2} \leq \sum_{\gamma_{j}^{2} \geq \frac{10}{9} \gamma_{n}^{2}} \frac{10}{\gamma_{j}^{2}} \ll \frac{\log ^{2} \gamma_{n}}{\gamma_{n}} .
$$

The sum $S_{1}$ is finite with largest term being the first, which is

$$
\frac{1}{\left(\gamma_{n+1}+\gamma_{n}\right)\left(\gamma_{n+1}-\gamma_{n}\right)} \ll \frac{\gamma_{n}^{\theta}}{2 \gamma_{n}} .
$$

The number of terms is bounded by

so

$$
\int_{\gamma_{n}}^{\sqrt{10 / 9} \gamma_{n}} \log t d t=O\left(\gamma_{n} \log \gamma_{n}\right)
$$

and therefore by $(2)$

$$
S_{1} \ll \gamma_{n}^{\theta} \log \gamma_{n}
$$

$$
-\log Y_{n} \ll \gamma_{n}^{2}\left(\gamma_{n}^{\theta} \log \gamma_{n}+\frac{\log ^{2} \gamma_{n}}{\gamma_{n}}\right) \ll \gamma_{n}^{2+\theta} .
$$

It follows from (1) and (3) that

$$
\log T_{n} \ll-\log \left|u_{t}\left(\frac{1}{2}, \gamma_{n}\right)\right| \ll \gamma_{n}^{2+\theta} .
$$

Gonek [11] defines

$$
\Theta=\inf \left\{\theta:\left|\zeta^{\prime}\left(\frac{1}{2}+i \gamma_{n}\right)\right|^{-1}=O\left(\left|\gamma_{n}\right|^{\theta}\right) \text { for all } n\right\} .
$$

Then [12] the Riemann Hypothesis implies $\Theta \geq 0$ and the averaged Mertens hypothesis (17, Section 14.29]) implies $\Theta \leq 1$. Gonek, on the basis of an analogy with eigenvalue statistics for random unitary matrices, conjectures $\Theta=1 / 3$. Hughes, Keating and O'Connell [12, following Gonek, surmise that $\Theta=1 / 3$ is in line with Montgomery's pair correlation conjecture [14, 16, which suggests $\Theta \geq 1 / 3$. To summarise this heuristic argument, the pair correlation conjecture 14 statement is that for fixed $a, b$ with $0<a \leq b<\infty$, as $T \rightarrow \infty$,

$$
\frac{\left|\left\{\left(\gamma, \gamma^{\prime}\right): 0<\gamma, \gamma^{\prime} \leq T, \frac{2 \pi a}{\log T} \leq \gamma-\gamma^{\prime} \leq \frac{2 \pi b}{\log T}\right\}\right|}{T \log T /(2 \pi)} \sim \int_{a}^{b}\left(1-\left(\frac{\sin \pi x}{\pi x}\right)^{2}\right) d x .
$$

Then $a$ is set to 0 and $b$ small so that the integral may be approximated by $\pi^{2} b^{3}$. Even though the conjecture is true for $T \rightarrow \infty$, assuming it holds for "small" $T$ leads to the inequality $b \gg T^{-1 / 3}$ so $\gamma_{n+1}-\gamma_{n} \gg \gamma_{n}^{-1 / 3}$. Finally assuming the reverse of an argument similar to that used in Theorem 4.3 below holds, we are led to suppose $\Theta \gg 1 / 3$.

Here we assume the Riemann Hypothesis and just $\Theta<\infty$, namely, that there exists a $\theta \geq 0$ such that

$$
\left|\zeta^{\prime}\left(\frac{1}{2}+i \gamma_{n}\right)\right|^{-1}=O\left(\left|\gamma_{n}\right|^{\theta}\right)
$$

for all $n \in \mathbb{N}$. We call this assumption WG or weak Gonek. 
Theorem 4.3. Assume the Riemann Hypothesis and WG. Then for all $n$

$$
\log T_{n} \leq \frac{\pi}{4} \gamma_{n}+O\left(\log \gamma_{n}\right) .
$$

Proof. Using Stirling's approximation to the Gamma function with $\rho_{n}=\frac{1}{2}+i \gamma_{n}$,

$$
\begin{aligned}
\log T_{n} & =\log 2 \pi-\log \left|\xi^{\prime}\left(\frac{1}{2}+i \gamma_{n}\right)\right| \\
& =\log 2 \pi-\log \left|\frac{\rho_{n}\left(\rho_{n}-1\right)}{2}\right|-\log \left|\pi^{-\rho_{n} / 2}\right|-\log \left|\Gamma\left(\rho_{n} / 2\right)\right|-\log \left|\zeta^{\prime}\left(\rho_{n}\right)\right| \\
& =-\log \left|\Gamma\left(1 / 4+i \gamma_{n} / 2\right)\right|-\log \left|\zeta^{\prime}\left(\rho_{n}\right)\right|+O\left(\log \gamma_{n}\right) \\
& =\frac{\pi}{4} \gamma_{n}+\log \left(1 /\left|\zeta^{\prime}\left(\rho_{n}\right)\right|\right)+O\left(\log \gamma_{n}\right),
\end{aligned}
$$

and the result follows.

The final equation in this proof can then be used to show

Corollary 4.1. Assume the Riemann Hypothesis. Then WG is true if and only if

$$
\log T_{n} \leq \frac{\pi}{4} \gamma_{n}+O\left(\log \gamma_{n}\right) .
$$

Theorem 4.4. Assume the Riemann Hypothesis. Then if WG is true there exists $\theta_{1}>0$ such that, for all $n \in \mathbb{N}$,

$$
\gamma_{n+1}-\gamma_{n} \gg \frac{1}{\gamma_{n}^{\theta_{1}}}
$$

Proof. Expand $f(t)=\zeta\left(\frac{1}{2}+i t\right)$, using Taylor's theorem about $\gamma_{n}$ as far as $\gamma_{n+1}$. There is an $\eta$ between $\gamma_{n}$ and $\gamma_{n+1}$ such that

$$
f\left(\gamma_{n+1}\right)=f\left(\gamma_{n}\right)+i f^{\prime}\left(\gamma_{n}\right)\left(\gamma_{n}-\gamma_{n+1}\right)-\frac{1}{2} f^{\prime \prime}(\eta)\left(\gamma_{n}-\gamma_{n+1}\right)^{2},
$$

so $\gamma_{n+1}-\gamma_{n}=2\left|f^{\prime}\left(\gamma_{n}\right)\right| /\left|f^{\prime \prime}(\eta)\right|$.

By Lemma 3.4

$$
\left|\zeta^{\prime \prime}\left(\frac{1}{2}+i \eta\right)\right| \ll \eta^{1 / 4} \log ^{4} \eta \leq \gamma_{n+1}^{1 / 4} \log ^{4} \gamma_{n+1} \ll \gamma_{n}^{1 / 4} \log ^{4} \gamma_{n} .
$$

Hence

$$
\gamma_{n+1}-\gamma_{n} \gg \frac{1}{\gamma_{n}^{\theta} \gamma_{n}^{1 / 4} \log ^{4} \gamma_{n}} \gg \frac{1}{\gamma_{n}^{\theta+1 / 4+\epsilon}},
$$

so we can choose $\theta_{1}=\theta+1 / 4+\epsilon$.

\section{Hilbert-Polya conjecture}

The well-known approach to proving the Riemann Hypothesis, attributed to Polya and Hilbert [15], is that there is a naturally occurring Hermitian operator whose eigenvalues are the zeros of $\xi\left(\frac{1}{2}+i t\right)$, which are therefore real [7, Page 345]. There have been many attempts to find such an operator [1, but none so far has been completely successful. The normal interpretation of the heights of the zeros $\left\{\gamma_{n}: n \in \mathbb{N}\right\}$ is that they correspond to frequencies of some, to be determined, vibrating system.

Consideration of the phase portraits described in [5, 6] in the light of the constancy of the period for rotation about each simple zero on the critical line [3, 
Theorem 2.3], gives rise to the notion that an alternative set of potential eigenvalues might be related to these periods $\left\{T_{n}: n \in \mathbb{N}\right\}$ in the usual manner:

$$
f_{n}=\frac{1}{T_{n}}=\frac{\lambda_{n}}{2 \pi}=\frac{\left|u_{t}\left(\frac{1}{2}, \gamma_{n}\right)\right|}{2 \pi} \approx \frac{e^{-\frac{\pi \gamma_{n}}{4}}}{2 \pi}
$$

so $\lambda_{i} \approx e^{-\frac{\pi \gamma_{i}}{4}}$. This approach has the following features:

(a) The eigenvalues have a natural relationship to $\zeta(s)$.

(b) The eigenvalues are the natural frequencies occurring in the flow $\dot{s}=\xi(s)$.

(c) The lowest zero corresponds to the largest eigenvalue and therefore to the mode of highest energy and largest frequency. This is quite natural, since the values of $\xi(s)$ with $s$ real and with values which are large and positive might be regarded as exerting a strong attractive pull on the flow, and would be expected to generate the greatest energy in the closest pair of zeros, in which corresponding frequencies become the "fundamental" modes. This "attractor" concept is strongly reinforced by the structure of the separatrices which all tend to the real axis [5, Theorem 3.2]. This is much more satisfactory than the normal approach in which the energy increases as the distance away from the real axis increases.

\section{ACKNOWLEDGMENTS}

The support of the University of Waikato Department of Mathematics and the helpful coments of Peter Sarnak and Steve Gonek are warmly acknowledged.

\section{REFERENCES}

[1] Berry, M.V. and Keating, J.P. The Riemann zeros and eigenvalue asympototics. SIAM Review 41 (1999), 236-266. MR1684543 (2000f:11107)

[2] Borwein, P. An efficient algorithm for the Riemann zeta function, Constructive, experimental and nonlinear analysis (Limoges, 1999), CMS Conf. Proc. 27 (2000), 29-34. MR.1777614 (2001f:11143)

[3] Broughan, K. A. Holomorphic flows on simply connected subsets have no limit cycles. Meccanica 38 (2003), 699-709. MR2028269 (2004m:37092)

[4] Broughan, K. A. and Barnett, A. R. Holomphic flow of the Riemann zeta function. Math. Comp. 73 (2004), 987-1004. MR2031420 (2004j:11092)

[5] Broughan, K. A. Holomorphic flow of Riemann's function $\xi(s)$. Nonlinearity 18 (2005), 12691294. MR 2134894

[6] Broughan, K.A. Phase portraits of the Riemann xi function zeros. http://www.math.waikato.ac.nz/ kab

[7] Conrey, J.B. The Riemann Hypothesis. Notices AMS 50 (2003), 341-353. MR.1954010 (2003j:11094)

[8] Edwards, T.M. Riemann's zeta function. Dover, New York, 1974. MR.1854455|(2002g:11129)

[9] Fujii, A. On the difference between $r$ consecutive ordinates of the Riemann Zeta function, Proc. Japan Acad. 51 (1975), 741-743. MR0389781 (52:10612)

[10] Godfrey, P. An efficient algorithm for the Riemann zeta function, http://www . mathworks.com/support/ftp zeta.m, etan.m (2000)

[11] Gonek, S.M. The second moment of the reciprocal of the Riemann zeta function and its derivative, lecture at the Mathematical Sciences Research Institute, Berkeley (June 1999).

[12] Hughes, C.P., Keating, J.P. and O'Connell, N. Random matrix theory and the derivative of the Riemann zeta function, Proceedings of the Royal Society: A 456 (2000), 2611-2627. MR.1799857 (2002e:11117)

[13] Levinson, N. and Montgomery, H. L. Zeros of the derivatives of the Riemann zeta function. Acta Math. 133 (1974), 49-65. MR0417074 (54:5135)

[14] Montgomery, H. L. The Pair correlation of zeros of the zeta function. Analytic Number Theory, Proceedings of the Symposia in Pure Mathematics 24 (1973), 181-193. MR0337821 $(49: 2590)$ 
[15] Odlyzko, A. M. Correspondence about the origins of the Hilbert-Polya conjecture. http://www.dtc.umn.edu/ oldyzko/polya/

[16] Oldyzko, A. M. On the distribution of spacings between zeros of the zeta function. Math. Comp. 48 (1987), 273-308. MR0866115 (88d:11082)

[17] Titchmarsh, E.C. as revised by Heath-Brown, D.R. The theory of the Riemann Zeta-function. Oxford, Second Ed., 1986. MR0882550 (88c:11049)

Department of Mathematics, University of Waikato, Hamilton, New Zealand

E-mail address: kab@waikato.ac.nz

Department of Mathematics, University of Waikato, Hamilton, New Zealand

E-mail address: arbus@math.waikato.ac.nz 